\title{
Cannabis for rheumatic pain: hope or hype?
}

\author{
Glen S. Hazlewood ${ }^{1,2,3,4}$ (D) Omid Zahedi Niaki ${ }^{5} \cdot$ Mary-Ann Fitzcharles $^{5,6,7}$
}

Received: 14 June 2019 /Revised: 19 June 2019 / Accepted: 21 June 2019/Published online: 5 July 2019

(C) International League of Associations for Rheumatology (ILAR) 2019

Even when inflammatory rheumatic diseases are "well controlled" by current standards, most patients continue to suffer ongoing pain, as well as other concomitant symptoms such as sleep disturbance, difficulty with mood, and impaired overall health [1]. Moreover, in the absence of any disease-modifying treatment for osteoarthritis, a condition likely to afflict most persons in the later years of life, chronic pain remains a prevailing symptom. The prevalence estimates for chronic pain in populations is in the order of $31 \%$ [2]. Chronic pain is often due to musculoskeletal conditions, with limited effectiveness and/or high rates of adverse events related to current pharmacological treatments. There is therefore an urgent need to identify new treatment strategies to alleviate pain. Could medical cannabis fill this void?

The hope that the plant Cannabis could be a therapeutic option for an array of medical conditions can be understood for several reasons. Preclinical study of the mammalian endocannabinoid system has pointed to an important function in maintaining physiologic equilibrium $[3,4]$. This encompasses not only effects on pain and inflammation but also

\section{Glen S. Hazlewood}

gshazlew@ucalgary.ca

Mary-Ann Fitzcharles

mary-ann.fitzcharles@muhc.mcgill.ca

1 Departments of Medicine and Community Health Sciences, Cumming School of Medicine, University of Calgary,

Calgary, Canada

2 Arthritis Research Canada, Calgary, Canada

3 McCaig Institute for Bone and Joint Health, Cumming School of Medicine, University of Calgary, Calgary, Canada

4 Cumming School of Medicine, University of Calgary, 3280 Hospital Dr NW, Calgary, Alberta T2N4Z6, Canada

5 Division of Rheumatology, McGill University Health Centre, Quebec, Canada

6 Alan Edwards Pain Management Unit, McGill University Health Centre, Quebec, Canada

7 Montreal General Hospital, McGill University Health Centre, 1650 Cedar ave, Montreal, Quebec H3G 1A4, Canada more diverse effects on neuronal maturation, sleep, appetite, and mood. However, due to the previous illegal status of Cannabis, the translation of preclinical studies into the clinical research arena has been both hindered and delayed [5]. This has resulted in a paucity of well-designed clinical studies and has opened the door for a proliferation of anecdote in both the medical literature as well as social media. The hype afforded by extensive media coverage in the last two decades has promoted the concept of a harmless panacea for many medical conditions ranging from severe neurological disease, cancer, mood disorder, substance abuse, and importantly pain. With lifting of prohibition in many jurisdictions, access to the herbal product as a modern-day medical therapy is now available. Sadly, Cannabis has been projected into this therapeutic window without following evidence-based standards for the evaluation for a medicinal product [5].

The groundswell of access to Cannabis began with legalization of medical cannabis in California in 1996, and thereafter, medical legalization in many countries worldwide. Uruguay and Canada are unique as the only sovereign states that have fully legalized the sale and consumption of recreational cannabis. Although medical cannabis has been available in Canada since 2001, a 2018 survey of Canadian rheumatologists reported that physicians lacked confidence in their knowledge of cannabinoids and their ability to counsel patients competently [6]. Ninety percent sought guidance in this regard, with most rheumatologists receiving daily questions from patients. Cannabinoids hold promise for rheumatology patients in view of the potential effects on pain and inflammation, the underpinnings of many rheumatic complaints. With more liberal access to Cannabis, it can be understood that patients may be requesting information about use, may be requesting prescriptions for use, or may even be experimenting with self-medication.

As a first step, rheumatologists must critically evaluate the evidence for effect of cannabinoids in order to provide evidence-based guidance for patients. The published literature in this regard can be confusing and unfortunately, the present state of evidence is poor for several reasons [7]. Many studies examining the effect of cannabinoids have included 
heterogenous patient populations with different pain conditions, rather than focusing on a defined condition. Studies are often of short duration, with some reviews even including studies of only 1-day duration. Thirdly, many reviews have been less meticulous in methodology, have not included metaanalyses, have not included unpublished studies, and have included all forms of cannabinoid treatments, without differentiating between the herbal product with varying routes of administration and pharmaceutical grade products. Therefore, the discrepant conclusions of various reviews can be explained by the thoroughness of the methodology applied. In a high-quality 2018 systematic review of the evidence for efficacy and safety of all cannabinoids for all chronic noncancer pain, including all study designs, the authors reported that $30 \%$ reduction in pain for cannabinoid groups vs. placebo was $29.0 \%$ vs. $25.9 \%$, with a number needed to benefit (NNTB) 24 (95\% CI 15-61) and number needed to harm (NNTH) 6 (95\% CI 5-8) [8]. The authors concluded that it is unlikely that cannabinoids are highly effective for chronic pain relief and there was minimal evidence for improving emotional or physical functioning.

With specific focus on rheumatic diseases, the evidence is even less convincing, with not a single study examining the effect of herbal cannabis in rheumatic diseases. In four randomized controlled trials of pharmaceutical cannabinoids with a total of 159 rheumatology patients (71 fibromyalgia, 30 spinal pain, 58 rheumatoid arthritis), with study duration up to five weeks, there was inconsistent evidence for superiority of cannabinoids over controls, noting that cannabinoids were generally well tolerated, but with some troublesome side effects. The authors concluded that there was insufficient evidence for recommendation of any cannabinoid preparation for symptom management in rheumatic diseases [9]. In contrast to the lack of evidence for benefit, all reviews report an increased rate of adverse events, mainly short-lived central nervous system effects such as dizziness, sleepiness, or fatigue for all cannabinoid preparations $[8,10]$. Importantly though, more serious adverse events such as psychosis have also been reported.

Regarding the data, a few key points need to be made. First, the lack of evidence of benefit should not be interpreted as evidence of a lack of benefit; we simply do not know. In practice patients are using Cannabis and frequently attest to its beneficial effects. Long-term adverse events in patient populations are unknown as randomized trials are typically limited to short-term data. On face value, the simplistic approach would be to recommend against use of any cannabinoid for rheumatology patients. However, a simple "no" may do little to help patients, who may choose to use it against medical advice or seek access from some other source and even via the "black market". Cannabis is a big industry, and guidance from practitioners linked to dedicated cannabis clinics have a conflict, whether real or perceived. Therefore, the medical community is in a quandary: on the one hand, Cannabis is strongly popularized as a neglected, but valuable therapy, and on the other hand, there remains a paucity of sound clinical data that can inform clinical care.

Rheumatologists and other health care practitioners are in a trusted position to listen to patients' questions and provide guidance. It is in this spirit of empathetic and realistic patient care that the Canadian Rheumatology Association has developed a pragmatic position statement for the use of medical cannabis for rheumatology patients [11]. This statement has focused on the herbal product, as in the preparations of flowers and leaves, either as the dried product or as an extraction of oils.

The essence of the position statement is that rheumatologists must remain the gatekeepers of clinical care for their patients; maintain a respectful, non-judgemental, and empathetic therapeutic relationship; and ensure the safety of patients and society. It is acknowledged that patients will be seeking advice about medical cannabis and may even be self-medicating. It must be emphasized that medical cannabis is not an alternative to standard care for any rheumatic disease or symptom related to the disease and physicians should adhere to current treatment standards and guidelines for rheumatic disease management. Although the efficacy of cannabis is unknown, some patients may truly benefit from its use. Patients should be informed of the risks and benefits of medical cannabis according to current evidence-based standards and physicians should not take recourse to anecdote. An understanding of the various cannabinoids is necessary: those produced within the organism are termed endocannabinoids, whereas exogenous cannabinoids are available as phytocannabinoids derived from the plant Cannabis, or as pharmaceutical-grade cannabis preparations that might be plant derived or synthesized.

Cautions for cannabis use were stated, including recommendations against use for those younger than 25 years, in view of the effect of cannabinoids on brain development, patients with allergy to cannabis, patients during pregnancy and breast feeding, and patients with history of psychosis, substance abuse, suicide ideation, or suicide attempts. An important aspect of this position statement is to highlight areas where caution should be exercised pending further clinical evidence. Particular attention should be given to the elderly in view of potential cognitive and psychomotor effects, with risks of an additive effect with psychoactive drugs. Confusion and falls may lead to harm. Psychomotor effects may impair function in all ages, with known impairment lasting for at least $5 \mathrm{~h}$ in experimental studies, and likely more prolonged. This is relevant to competence to drive, with increasing evidence that cannabis is a risk for motor vehicle accidents and road fatalities [12]. Similarly, caution was recommended for cannabis use in any work setting requiring concentration, optimal executive function, and alertness. The emerging evidence for 
cardiovascular risks is relevant for rheumatology patients. Although not reported for clinical trials, epidemiological reports of cardiovascular events in the community associated with cannabis use are notable.

The reality is that Cannabis is now entrenched in medical care and will continue to be used by many patients. As rheumatologists, we need to listen to our patients and be empathetic and pragmatic in our approach, while remaining rooted in the current best available evidence. We should counsel patients who choose to use Cannabis on the current lack of evidence for effect in rheumatic diseases and ensure that patients are informed of the known side effects and unknown long-term harms. We must advocate for well-designed clinical studies to evaluate the benefits and risks of cannabis as an adjunct therapy for rheumatic pain. This research is needed to truly understand whether cannabinoids in general will hold hope for patients suffering from chronic pain.

Acknowledgments Glen Hazlewood is supported by a CIHR New Investigator Salary Award.

\section{Compliance with ethical standards}

Disclosures None.

\section{References}

1. Ishida M, Kuroiwa Y, Yoshida E, Sato M, Krupa D, Henry N, Ikeda K, Kaneko Y (2018) Residual symptoms and disease burden among patients with rheumatoid arthritis in remission or low disease activity: a systematic literature review. Mod Rheumatol 28(5):789-799. https://doi.org/10.1080/14397595.2017.1416940

2. Steingrimsdottir OA, Landmark T, Macfarlane GJ, Nielsen CS (2017) Defining chronic pain in epidemiological studies: a systematic review and meta-analysis. Pain 158(11):2092-2107. https://doi. org/10.1097/j.pain.0000000000001009

3. Anand P, Whiteside G, Fowler CJ, Hohmann AG (2009) Targeting $\mathrm{CB} 2$ receptors and the endocannabinoid system for the treatment of pain. Brain Res Rev 60(1):255-266. https://doi.org/10.1016/j. brainresrev.2008.12.003

4. Mechoulam R, Hanus LO, Pertwee R, Howlett AC (2014) Early phytocannabinoid chemistry to endocannabinoids and beyond. Nat Rev Neurosci 15(11):757-764. https://doi.org/10.1038/nrn3811

5. D'Souza DC, Ranganathan M (2015) Medical marijuana: is the cart before the horse? JAMA 313(24):2431-2432. https://doi.org/10. 1001/jama.2015.6407

6. Therapeutics (2017) Survey results on medical marijuana. The Journal of the Canadian Rheumatology Association 27(4):27

7. Hauser W, Finnerup NB, Moore RA (2018) Systematic reviews with meta-analysis on cannabis-based medicines for chronic pain: a methodological and political minefield. Pain. 159:1906-1907. https://doi.org/10.1097/j.pain.0000000000001295

8. Stockings E, Campbell G, Hall WD, Nielsen S, Zagic D, Rahman R, Murnion B, Farrell M, Weier M, Degenhardt L (2018) Cannabis and cannabinoids for the treatment of people with chronic noncancer pain conditions: a systematic review and meta-analysis of controlled and observational studies. Pain 159(10):1932-1954. https://doi.org/10.1097/j.pain.0000000000001293

9. Fitzcharles MA, Baerwald C, Ablin J, Hauser W (2016) Efficacy, tolerability and safety of cannabinoids in chronic pain associated with rheumatic diseases (fibromyalgia syndrome, back pain, osteoarthritis, rheumatoid arthritis): a systematic review of randomized controlled trials. Schmerz (Berlin, Germany) 30(1):47-61. https:// doi.org/10.1007/s00482-015-0084-3

10. Nugent SM, Morasco BJ, O'Neil ME, Freeman M, Low A, Kondo K, Elven C, Zakher B, Motu'apuaka M, Paynter R, Kansagara D (2017) The effects of Cannabis among adults with chronic pain and an overview of general harms: a systematic review. Ann Intern Med 167(5):319-331. https://doi.org/10.7326/m17-0155

11. Fitzcharles MA, Niaki OZ, Hauser W, Hazlewood G (2019) Position statement: a pragmatic approach for medical Cannabis and patients with rheumatic diseases. J Rheumatol 46(5):532538. https://doi.org/10.3899/jrheum.181120

12. Asbridge M, Hayden JA, Cartwright JL (2012) Acute cannabis consumption and motor vehicle collision risk: systematic review of observational studies and meta-analysis. BMJ 344:e536. https://doi.org/10.1136/bmj.e536

Publisher's note Springer Nature remains neutral with regard to jurisdictional claims in published maps and institutional affiliations. 